\title{
Electromagnetic Near Field Interference with Implantable Medical Devices
}

\author{
Roger Carrillo, MD, Mount Sinai Hospital, Miami, Florida \\ Oscar Garay, EE, Motorola, Plantation, Florida \\ Q. Balzano, Ph.D., Motorola, Plantation, Florida \\ Michael Pickels, EE, Mount Sinai Hospital, Miami, Florida
}

\begin{abstract}
The paper describes the methodology used to detect interference phenomena in vitro caused by digital and analog cellular phones placed in the close vicinity of implantable cardiac pacemakers. All four pacemakers tested exhibited some kind of interference; there was inhibition of the pacemaker function in two devices, when these were located at less than 2.5 $\mathrm{cm}$ from the antenna. The other two pacemakers exhibited atrial tracking of the interfering electromagnetic energy when exposed in similar situation.
\end{abstract}

\section{INTRODUCTION}

Theoretical considerations of electromagnetic energy density near commonly available digital portable cellular phones [1] show that in some conditions it is possible to expose implanted cardiac pacemakers to magnetic field intensities higher than that of a $10 \mathrm{~mW} / \mathrm{cm}^{2}$ plane incident wave, for which these devices have designed interference immunity. Preliminary results from other authors [2-3] using European digital telephones near implanted cardiac pacemakers in vivo and in vitro have detected a variety of interference phenomena including inhibition. This study was performed to test if cellular telephones employing the American digital signaling systems of cellular telephony cause cardiac pacemaker interference in vitro, when placed in the immediate vicinity of the implantable medical devices.

\section{MATERIALS AND METHODS}

Seven different types of cellular telephones have been tested during the study. They operate in the frequency range of 800 $900 \mathrm{MHz}$. Two analog phones with continuous wave (CW) FM transmitter had $0.6 \mathrm{~W} \quad \mathrm{RF}$ output power to the antenna. Two phones with the North American Digital Cellular signaling system transmitted $50 \mathrm{~Hz}$ pulses of $6.6 \mathrm{~ms}$ duration and $0.6 \mathrm{~W}$ RF power. Two phones with the European signaling system (GSM) emitted $217 \mathrm{~Hz}$ pulses of $0.576 \mathrm{~ms}$ duration and $2 \mathrm{~W} \mathrm{RF}$

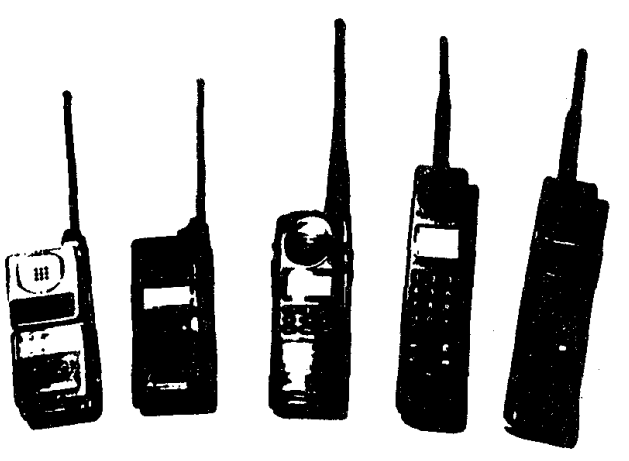

Figure 1: Cellular Phones Used for Tests power. The last phone transmitted $11 \mathrm{~Hz}$ pulses of $15 \mathrm{~ms}$ duration and about $2 \mathrm{~W}$ average power during each pulse. A picture of the phones emitting RF pulsed is shown in figure 1 . The $\mathrm{CW}$ phones have the same outward shape as the first and the fourth device from the left in figure 1.

The artificial chest used in test protocol is a clear plastic container of $30 \mathrm{~cm} \times 20 \mathrm{~cm} \times 14 \mathrm{~cm}$ size filled with a $0.9 \%$ (in weight) saline solution. The experimental setup is shown in figure 2. In absence of the pacemaker the set up was tested for absence of detectable interference signals displayed on the oscilloscope of the ECG monitoring equipment (Electronics for Medicine Inc. Model IM Bedside Monitor, manufacturer serial number 106862), when the active RF sources were placed at the test location.

\section{EXPERIMENTAL SETUP}

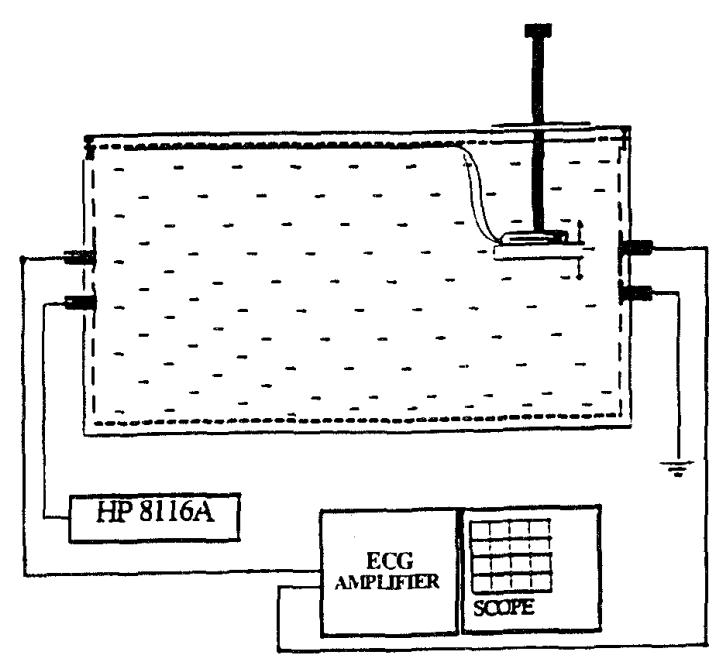

Figure 2

The near magnetic and electric fields inside the artificial chest were recorded in presence of the phone but in absence of the pacemaker. The near $\mathrm{E}$ - and $\mathrm{H}$ - fields of the phones inside the artificial chest were measured by employing instrumentation described in other works [4-5]. Finally the pacemaker was located at about $1-2 \mathrm{~cm}$ depth in the saline solution, as shown in figure 2. Three (3) DDD and one VVI pacemakers were used in the test program and exposed to the cellular phones which were positioned by means of a Plexiglas holder overhanging the saline bath. The holder could be raised or lowered above the water level in the artificial chest. 


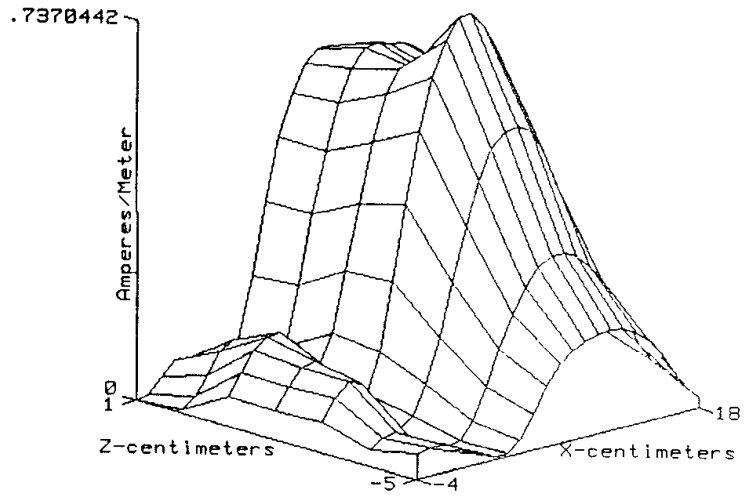

Figure 3: H-field Amplitude Measured in Saline Solution

\section{RESULTS}

For reasons of brevity only the highlights of the results will be presented herein. Typical maps of the near magnetic and electric fields sustained by one of the phones at about $3 \mathrm{~cm}$ from the axis of its antenna, that is about $1 \mathrm{~cm}$ inside the saline solution in absence of the pacemaker, are shown in figures 3 and 4. Please note that the shape of the electric and magnetic field distribution is approximately the same showing that the electric field inside the test chest is induced by the magnetic field which penetrates the saline solution without substantial attenuation because of its low characteristic impedance [1 and 6].

Different pacemakers showed various degrees of susceptibility to the interfering EM signals. In general, the FM transmitters caused no significant interference phenomena. The interference of relevance was noticed with the cellular phones which emit pulsed signals. All pacemakers had some sensitivity to the pulsing $R F$ energy ranging from atrial tracking to outright inhibition. The worst cases of interference were noticed when the plastic header of the cardiac pacemaker was located at or near the peak of the electric field inside the saline solution.

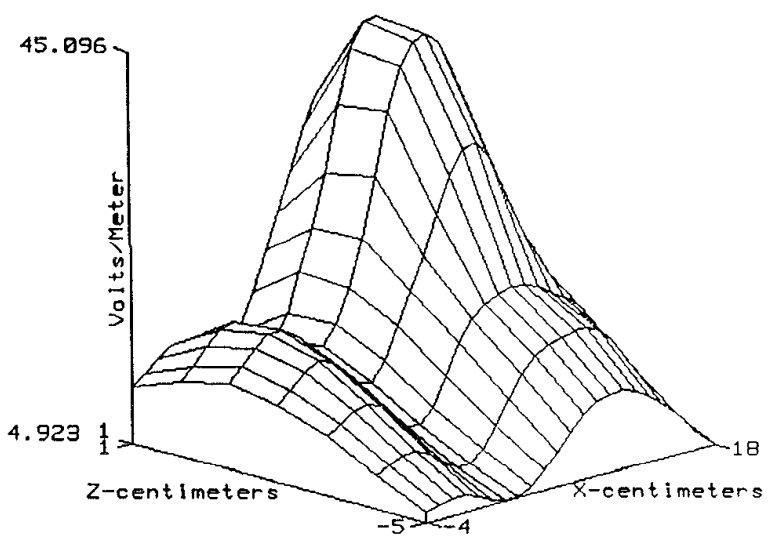

Figure 4: E-field Amplitude Measured in Saline Solution
The situation is depicted in figure 5 , showing a topographic map of the field distribution of figure 4 with the silhouette of a pacemaker outlined in the condition of worst interference condition. Note that there are no leads connected to the pacer in the figure. The length of the leads has minimal impact on the interference phenomena, which appear to be caused by a direct interaction between the EM fields and the region of the header of the pacemaker. Figure 5 also shows the clear cut case of pacing inhibition detected in this situation and recorded using the printer of the monitor unit. The distance of the phones from the surface of the saline solution at which the interference phenomena are detected varies between $2 \mathrm{~cm}$ and about $10 \mathrm{~cm}$ for the various combinations of phone-pacemaker.
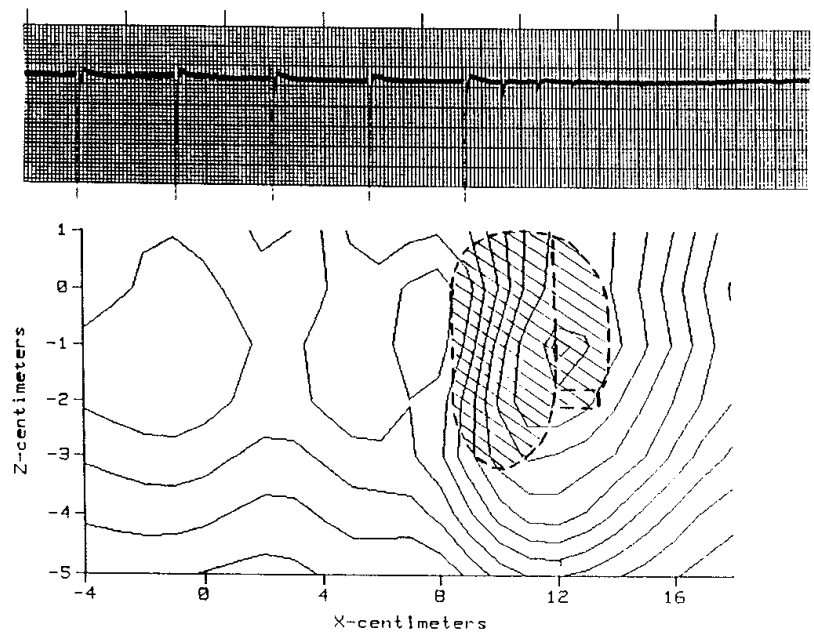

Figure 5: Position of Pacemaker in the Interfering Field Map

\section{CONCLUSION}

From the preliminary results presented herein, it has been shown that the pacemaker leads are not important in the interference phenomena detected near portable cellular phones. The pacers are most sensitive when the peak of the electric (and magnetic) energy inside the test bath is close to the device headers.

Finally, the evaluation of electromagnetic interference in pacemakers testing should include the electromagnetic near fields of cellular telephones because of the large proliferation of these devices and their ability of producing interaction with medical implantable devices.

\section{REFERENCES}

[1] Q. Balzano, "Considerations on the Interference Phenomena Near Resonant Antennas Emitting RF Pulses," Proceedings of the EMC Conference, Zurich, Switzerland, 3/7-3/9, 1995

[2] B. Eicher et al, "Effects of TDMA-Modulated Hand-Held Telephones on Pacemakers," presented at the Bioelectromagnetic Society Symposium, Copenhagen, Denmark, June 1994. 
[3] V. Barbaro et al, "GSM Cellular Phone Interference with Implantable Pacemakers: In Vitro and In Vivo Observation," presented at the Bioelectromagnetic Society Symposium, Copenhagen, Denmark, June, 1994.

[4] Q. Balzano et al, "Electromagnetic Energy Exposure of Simulated Users of Portable Cellular Phones," accepted for publication by the IEEE Vehicular Technology Society, In Print.

[5] T. J. Babij et al, "Measurement of the Magnetic Field Very Close to an RF Source," in Record of the IEEE Vehicular Technology Conference, Pittsburgh, PA, May 21-23, 1984, pp. 57-60.

[6] N. Kuster and Q. Balzano, "Energy Absorption Mechanism by Biological Bodies in the Near Field of Dipole Antennas Above $300 \mathrm{MHz}$, IEEE Transactions on Vehicular Technology, Vol. 41, No. 1, pp. 17-23, Feb. 1992. 